\title{
Desenvolvimento de uma ontologia para Recursos Educacionais Abertos e Fragmentados usando a abordagem METHONTOLOGY
}

\author{
Ubirajara Santos Nogueira ${ }^{1,2}$, Ricardo José Rocha Amorim³ ${ }^{3}$, Bernadette Farias \\ Lóscio ${ }^{1}$ \\ ${ }^{1}$ Centro de Informática - Universidade Federal de Pernambuco (UFPE) \\ Caixa Postal 7851 - 50.732-970 - Recife - PE - Brasil \\ ${ }^{2}$ Instituto Federal do Sertão Pernambucano (IFSertão-PE) - Campus Petrolina \\ BR 407, KM 08 - Jardim São Paulo - 56.314-520 - Petrolina - PE - Brasil \\ ${ }^{3}$ Faculdade de Ciências Aplicadas e Sociais de Petrolina (FACAPE) \\ Campus Universitário s/n - Vila Eduardo - 56.328-903 - Petrolina - PE - Brasil \\ usnecin.ufpe.br, amorim.ricardo@gmail.com, bflecin.ufpe.br
}

\begin{abstract}
The Open Educational Resources (OER) are learning objects available for any user to freely use. These resources are very useful for the learning process of students of distance learning and classroom teaching. OER should be available in specific repositories, under public domain or open licenses in order to facilitate its reuse. This paper describes the development of an ontology for the OER domain, which allows not only the representation of the OER as a whole, but also the representation of parts of the contents of resources. In order to guide the ontology development, a methodology, called Methontology, was adopted.
\end{abstract}

Resumo. Os Recursos Educacionais Abertos (REA) são objetos de aprendizagem disponibilizados para qualquer usuário usar livremente, sendo úteis para o aprendizado de alunos dos ensinos à distância e presencial. Os REA devem ser disponibilizados em repositórios específicos, sob domínio público ou licenciados de maneira aberta, de forma a facilitar seu reúso. Neste contexto, este trabalho descreve o desenvolvimento de uma ontologia para o domínio de REA, a qual permite não apenas a representação do REA como um todo, mas também a representação de partes do conteúdo dos recursos. Para guiar o processo de desenvolvimento da ontologia, uma metodologia, denominada Methontology, foi adotada.

\section{INTRODUÇÃO}

A característica principal de um Recurso Educacional Aberto (REA) é que esteja disponível para qualquer usuário, permitindo valorizar práticas de aprendizagem ligadas ao uso da Web e da sociedade do conhecimento, além de fortalecer o sujeito que produz o conteúdo e colocar o autor no centro das atenções [Santana et al. 2012]. Os REA devem estar sob domínio público ou licenciados de maneira aberta, por licenças de direito autoral livre, como o padrão Creative Commons, uma organização sem fins lucrativos voltada ao compartilhamento e uso da criatividade e do conhecimento por 
meio de instrumentos legais livres [Creative Commons 2014]. Por meio do uso dos REA, é possível contar com a reutilização de conteúdos de aprendizagem que, reaproveitados em artefatos digitais, podem melhorar a forma de entrega de um conteúdo educacional [Casali et al. 2013]. Para favorecer a reutilização, os REA devem estar disponíveis em um formato que seja entendido por máquinas e, além disso, devem ser disponibilizados de forma fragmentada, ou seja, o recurso não deve ser disponibilizado como um todo, mas sim em fragmentos [Santos 2013]. No caso de um REA do tipo livro, por exemplo, que é constituído de diversos capítulos, onde cada capítulo possui várias seções e cada seção é formada por parágrafos, figuras, tabelas e gráficos, cada um desses componentes do conteúdo do livro, deve ser disponibilizado separadamente. Para possibilitar a disponibilização de REA de forma fragmentada, modelos semânticos, como as ontologias, podem ser utilizados, facilitando o reúso dos fragmentos desejados por parte dos autores de novos REA [Casali et al. 2013].

Apesar do uso de ontologias ser muito comum em diversas áreas do conhecimento, construir ontologias não é uma tarefa fácil. Dentre os desafios encontrados na criação de ontologias, destacam-se problemas referentes a estruturação de dados [Mattos et al. 2010], falta de padronização na representação do conhecimento e dificuldades para a implementação da ontologia [Guimarães 2002]. Para auxiliar o desenvolvimento de ontologias, já foram propostas diversas metodologias, linguagens e ferramentas [Corcho et al. 2003], dentre as quais destaca-se a metodologia Methontology [Fernández-López et al. 1997]. Neste contexto, este artigo detalha o desenvolvimento da ontologia ONTOER + , que tem como principal objetivo permitir a representação do conteúdo de REA em fragmentos, facilitando, dessa forma, o seu reúso. A ontologia ONTOER+ foi desenvolvida a partir da ontologia ONTOER proposta por Amorim et al. (2012), que tem como base as especificações de metadados LOM (Learning Object Metadata) [Hodgins 2002] e DocBook [Walsh 1999]. Ainda é objetivo deste trabalho, demonstrar o uso da abordagem Methontology no desenvolvimento da ontologia ONTOER+.

O restante deste artigo está organizado da seguinte forma: a Seção 2 descreve o uso da metodologia Methontology na criação da ontologia ONTOER+; a Seção 3 apresenta um exemplo que ilustra como a ontologia ONTOER+ pode ser utilizada para representação de um REA do tipo Slide Show e a última seção apresenta as considerações finais.

\section{DESENVOLVIMENTO DA ONTOLOGIA ONTOER+ BASEADA NA ABORDAGEM METHONTOLOGY}

De maneira geral, o processo de desenvolvimento de ontologias segue o mesmo padrão utilizado para o desenvolvimento de software. Porém, do ponto de vista metodológico, um dos grandes problemas com o desenvolvimento de ontologias é que não há um padrão definido para a construção destas. Apesar de um grande número de ontologias já terem sido desenvolvidas por diferentes comunidades, utilizando abordagens, métodos e técnicas diferenciadas, não há consenso sobre uma metodologia para o processo de construção destes artefatos. Desta forma, há uma ausência de atividades padronizadas, as quais, em geral, são realizadas de forma artesanal e contrária a um padrão de desenvolvimento [Suárez-Figueroa et al. 2012]. Neste sentido, o trabalho proposto em [Silva et al. 2008] destaca as seguintes metodologias para construção de ontologias: metodologia para o projeto Tove [Fox 1992], Methontology [Fernández-López et al. 
1997] e On-To-Knowledge [Corcho et al. 2003]. Estas metodologias apresentam características e abordagens diversas, não sendo apontada a melhor dentre elas, uma vez que a escolha por uma metodologia dependerá do tipo de ontologia a ser desenvolvida, dentre outros fatores.

Para o desenvolvimento da ontologia ONTOER+, optou-se por fazer uso da metodologia Methontology, escolhida em função de ter sido indicada por vários autores e utilizada em vários trabalhos [Corcho et al. 2003], [Fernández-López et al. 1997], [Park et al. 2008] e [Bláquez 1998]. A Methontology vem sendo utilizada no desenvolvimento de vários projetos em diferentes domínios do conhecimento [Park et al. 2008], sendo composta por um conjunto de seis atividades: especificação, conceitualização, formalização, integração, implementação e manutenção [FernándezLópez et al. 1997], as quais serão descritas a seguir.

A atividade de especificação procura justificar porque a ontologia está sendo construída, qual o uso previsto e quem são os usuários. A conceitualização organiza e converte a especificação em um modelo conceitual com base em tabelas e gráficos. A atividade de formalização transforma o modelo conceitual em um modelo formal. Com o objetivo de acelerar a construção da ontologia, a atividade de integração, considera a reutilização de definições já construídas em outras ontologias, em vez de começar do zero. A atividade de implementação constrói modelos computáveis em uma linguagem de ontologias, como, OWL (Ontology Web Language). Finalmente, a atividade de manutenção prevê correções na ontologia. Com o intuito de ilustrar o processo de desenvolvimento da ontologia ONTOER+, neste trabalho, são detalhadas as atividades de especificação e conceitualização. Vale ressaltar que a ontologia já foi implementada em linguagem OWL e, atualmente, encontra-se em fase de manutenção, porém, por questões de espaço, não serão apresentados detalhes dessas atividades, Para representar os resultados produzidos em cada uma das etapas descritas acima, foram escolhidos os artefatos propostos em [Fernández-López et al. 1997].

\subsection{Especificação}

Conforme Silva (2009), a atividade de especificação tem como objetivo produzir um documento em linguagem natural definindo o escopo da ontologia e incluindo seus termos, características e granularidade. Para a ONTOER + , nesta fase, produziu-se um documento em linguagem natural, identificando os principais conceitos do domínio. $\mathrm{O}$ conhecimento foi adquirido tendo como base o padrão LOM [Hodgins 2002] e a especificação DocBook [Walsh 1999]. O LOM é o padrão de metadados para Objetos de Aprendizagem (OA), definido pelo Institute of Electrical and Electronics Engineers (IEEE), e tem como objetivo facilitar a pesquisa, aquisição, evolução e uso desses objetos [Hodgins 2002]. O DocBook é um conjunto de definições e regras para orientar a criação de documentos estruturados usando as tecnologias SGML ou XML, indicando como um documento DocBook válido deve ser estruturado, sendo bastante adequado para a escrita de livros e artigos [Walsh 1999]. O LOM foi muito útil para a definição do conceito de Learning Object, enquanto que o DocBook foi fundamental para a definição do conceito Publication, um dos tipos de OA considerados na ontologia ONTOER+. Outros tipos de OA, como apresentação em slides, tiveram seu conhecimento adquirido de forma ad hoc a partir de consultas a especialistas do domínio.

Como resultado da fase de conceitualização, foi criado o documento de especificação da ontologia, aqui apresentado resumidamente: 
- Objetivos: A ontologia tem como principal propósito a representação, de forma fragmentada, de recursos educacionais abertos do tipo publicação e apresentação em slides, sendo indicada para os casos em que busca-se aumentar a reutilização dos materiais de aprendizagem disponibilizados.

- Usuários: Apenas o autor do objeto fará uso da ontologia. Imaginemos um professor (autor) que confecciona uma determinada apostila para ser utilizada em suas aulas e deseja disponibilizá-la para seus alunos. Para isso, ele disponibiliza o conteúdo da apostila de forma fragmentada, permitindo, dessa forma, a sua utilização pelo grupo de alunos de sua disciplina ou qualquer outro usuário que deseje acessar a apostila inteira, apenas um capítulo ou até mesmo simplesmente um parágrafo.

- Escopo: A ontologia criada deverá permitir: (i) disponibilizar publicações (artigos, livros, apostilas e similares) de forma fragmentada: prefácio, indice, capítulo, seção, parágrafo, tabela, gráfico, figura, lista, nota de rodapé, palavras estrangeiras, listas de itens, apêndice e bibliografia; (ii) disponibilizar apresentações em slides de forma fragmentada: figura, tabela, itens, texto, título.

\subsection{Conceitualização}

Nesta etapa do desenvolvimento da ontologia ONTOER+, várias atividades foram desenvolvidas com o objetivo de melhor entender o domínio estudado. De acordo com Fernández-López et al. (1997), as seguintes atividades fazem parte da etapa de Conceitualização:

1. Identificar conceitos, instâncias, atributos e valores em um dicionário de dados.

2. Classificar os grupos de conceitos em Árvores de classificação de Conceitos.

3. Descrever constantes em uma Tabela de Constantes.

4. Descrever atributos de instância e atributos de classe nas tabelas de atributos de instância e tabelas de atributos de classe.

5. Descrever fórmulas utilizadas para inferir valores numéricos dos atributos usando tabelas de Fórmulas.

6. Inferir atributos nas Árvores de classificação de Atributos.

7. Descrever indivíduos em Tabelas de Instâncias.

Nas seções seguintes, são apresentadas cada uma das atividades desenvolvidas neste trabalho. Algumas tabelas sugeridas não foram criadas por não serem necessárias no domínio de aplicação da ONTOER+.

\subsubsection{Dicionário de Dados}

A tabela para descrição do Dicionário de Dados, com a identificação e apresentação de todos os conceitos do domínio potencialmente úteis, seus significados, atributos e instâncias, conforme sugerido por Gómez-Pérez et al. (1996), foi alterada com a inclusão da coluna origin, usada para identificar a origem do conceito. Para cada conceito identificado no domínio, deu-se o preenchimento da Tabela 1. Por motivo de espaço, apenas uma destas tabelas é apresentada parcialmente. Para ilustrar a definição de conceitos, considere a descrição do conceito Learning Object. Também foram identificados os conceitos Preface, Index, Chapter, Block, In Line Elements, Section, Figure, Graphic, List of Items, Paragraph, Table, Text, Title, Appendix, Glossary, Bibliography, Slide Show e Slide. 
Tabela 1. Apresentação parcial do dicionário de dados para um OA tipo Publication.

\begin{tabular}{|c|c|c|c|c|c|c|c|}
\hline $\begin{array}{c}\text { Concept } \\
\text { Name }\end{array}$ & Origin & Synonym & Acronym & Description & Instance & $\begin{array}{c}\text { Class } \\
\text { Attributes }\end{array}$ & Instance Attributes \\
\hline $\begin{array}{l}\text { Learning } \\
\text { Object }\end{array}$ & LOM & & LO & $\begin{array}{l}\text { Learning objects are } \\
\text { digital tools or } \\
\text { resources which may be } \\
\text { used and reused to } \\
\text { support learning. Such } \\
\text { resources may include } \\
\text { videos, images, } \\
\text { hypertext, ... }\end{array}$ & $\begin{array}{l}\text { Towards a } \\
\text { Method to } \\
\text { Conceptual } \\
\text { ize } \\
\text { Domain } \\
\text { Ontologies }\end{array}$ & & $\begin{array}{l}\text { Catalog-Of-Identifier-Of- } \\
\text { General } \\
\text { Entry-Of-Identifier-Of-General } \\
\text { Title-Of-General } \\
\text { Language-Of-General } \\
\text { Description-Of-General } \\
\text { Keyword-Of-General }\end{array}$ \\
\hline
\end{tabular}

\subsection{2 Árvore de Classificação de Conceitos}

As Árvores de Classificação de Conceitos organizam os conceitos do domínio em taxonomias. Elas são usadas não só para entender como os conceitos se relacionam entre si, mas para modularizar o conhecimento do domínio em ontologias independentes. Para cada conjunto disjunto de conceitos, uma árvore de classificação de conceitos é construída [Gómez-Pérez et al. 1996]. Após uma análise minuciosa dos conceitos encontrados durante a definição do dicionário de dados, os mesmos foram divididos em seis árvores: Learning Object, Components, Block, List e Slide-Show. O nível mais geral da árvore foi subdividido em duas grandes árvores Learning Object e Components, como podemos observar na Figura 1.

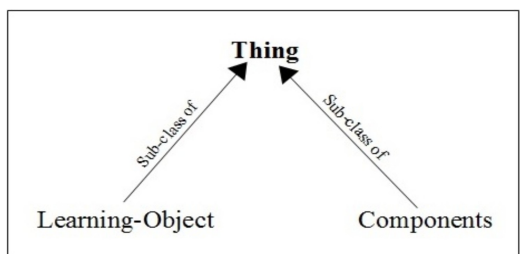

Figura 1. O nível mais geral da árvore de classificação de conceitos

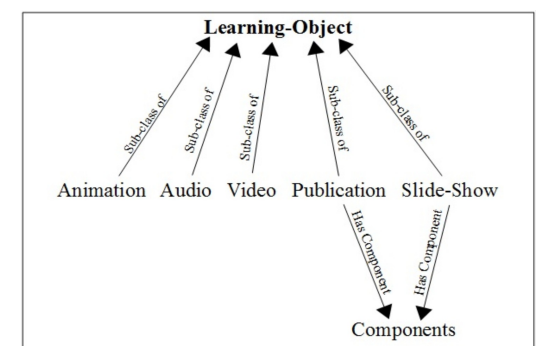

Figura 2. Árvore de classificação do conceito Learning-Object.

$\mathrm{Na}$ árvore Learning Object foram agrupados os conceitos identificados como OA propriamente ditos: Publication e Slide Show e possuem um conjunto de componentes específicos. A Figura 2 apresenta a árvore criada para Learning Object assim como a relação entre os Objetos Publication e Slide Show com o conceito Components. $\mathrm{Na}$ árvore de conceitos Components, demonstrada na Figura 3, foram agrupados os conceitos appendix, bibliography, block, chapter, glossary, index, in-lineelements, preface, section e slide. Estes conceitos são comumente encontrados em OA do tipo publicação e apresentação em slides e são considerados conceitos disjuntos.

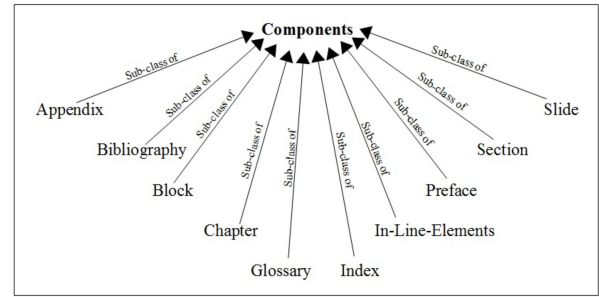

Figura 3. Árvore de classificação do conceito Components.

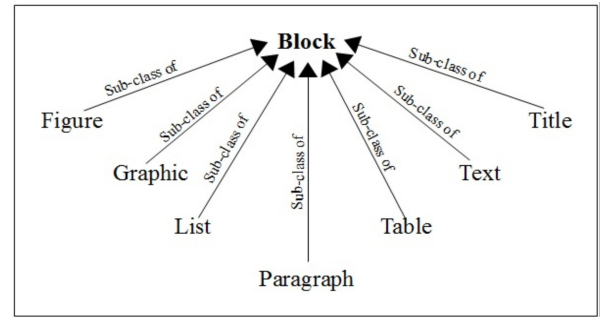

Figura 4. Árvore de classificação do conceito Block. 
$\mathrm{Na}$ árvore de conceitos chamada block (Figura 4), foram agrupados os conceitos relacionados a um bloco: figure, graphic, list, paragraph, table, text $\mathrm{e}$ title. Nesta árvore foram agrupados os conceitos que compõem um bloco dentro da estrutura de uma publicação ou apresentação em slide. A árvore list foi criada para agrupar os tipos de lista itemized-List e ordered-List, que são sub-conceitos do conceito list. A Figura 5 apresenta a árvore construída para o conceito list. A árvore slide show foi criada para apresentar o conceito Slide que é um sub-conceito do conceito Slide Show. A Figura 6 apresenta a árvore construída para o conceito Slide Show.

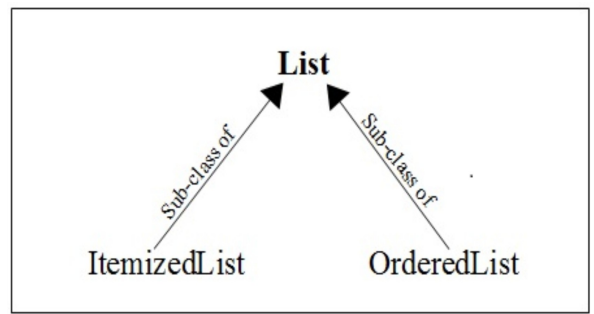

Figura 5. Árvore de classificação do conceito List.

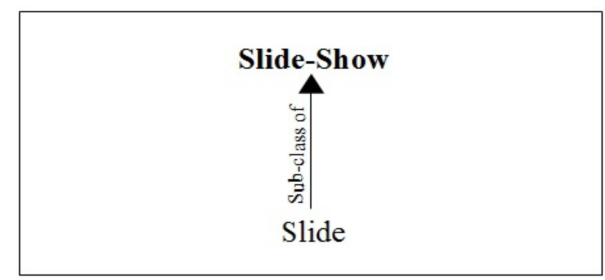

Figura 6. Árvore de classificação do conceito Slide Show.

\subsubsection{Tabelas de Atributos de Instância}

Os atributos de instância são assim definidos por [Guimarães 2002]: "Atributos de instância são os atributos definidos nos conceitos, mas que são valorados nas instâncias". Uma tabela de atributos de instância fornece informações sobre um atributo ou sobre os seus valores em uma instância. Para cada atributo de instância, descrito no Dicionário de Dados da ontologia ONTOER+, uma tabela foi criada, conforme orientação da metodologia. A Tabela 2 descreve parcialmente o atributo Difficulty-OfEducational definido no conceito Learning Objects.

Tabela 2. Tabela do atributo de instância Difficulty-Of-Educational

\begin{tabular}{|l|l|}
\hline Instance Attribute Name & Difficulty-Of-Educational \\
\hline Description & $\begin{array}{l}\text { How hard it is to work with or through this learning object for the typical intended target audience. } \\
\text { NOTE:--The "typical target audience" can be characterized by data elements Context-Of-Educational } \\
\text { and TypicalAgeRange-Of-Educational. }\end{array}$ \\
\hline Value type & String \\
\hline Range of values & very easy, easy, medium, difficult, very difficult \\
\hline
\end{tabular}

\subsubsection{Tabelas de Instâncias}

Uma tabela de instâncias descreve as instâncias do domínio através do nome, seus atributos de instância e valores para os mesmos. De acordo com o trabalho proposto em [Gómez-Pérez et al. 1996], os campos Instance Name, Description, Attributes e Values devem ser usados para descrever as instâncias. Por motivo de espaço, a Tabela 3 apresenta apenas um exemplo de instância do conceito Publication. 
Tabela 3. Tabela de Instâncias - Publication

\begin{tabular}{|c|c|c|c|}
\hline Instance Name & Description & Attibutes & Values \\
\hline \multirow{4}{*}{$\begin{array}{l}\text { Towards a } \\
\text { Method to } \\
\text { Conceptualize } \\
\text { Domain } \\
\text { Ontologies }\end{array}$} & \multirow{4}{*}{$\begin{array}{l}\text { This is an } \\
\text { instance of } \\
\text { the concept } \\
\text { Publication }\end{array}$} & Title-Of-General & Towards a Method to Conceptualize Domain Ontologies \\
\hline & & Language-Of-General & En \\
\hline & & $\begin{array}{l}\text { Description-Of- } \\
\text { General }\end{array}$ & $\begin{array}{l}\text { This paper presents the suite of principles, designs criteria and } \\
\text { verification process used in the knowledge conceptualization process of } \\
\text { a consensuated domain ontology in the domain of chemicals. To achieve } \\
\text { agreement between different development teams we propose the use of a } \\
\text { common and shared conceptual model as starting point. To capture } \\
\text { domain knowledge of a given domain and organize ... }\end{array}$ \\
\hline & & Keyword-Of-General & [ontology], [methodology], [methontology] \\
\hline
\end{tabular}

\section{CASO DE USO DA ONTOLOGIA ONTOER+}

Esta seção apresenta um exemplo de uso da ontologia ONTOER+, que ilustra o processo de instanciação de um determinado objeto de aprendizagem, com foco na relação entre o conteúdo a ser instanciado e a ontologia. A Figura 7 apresenta a ontologia ONTOER+ com recortes obtidos através da ferramenta Protégé. No lado esquerdo, é possível visualizar todas as classes da ontologia. $\mathrm{Na}$ região central é possível identificar algumas restrições relacionadas com as classes Slide, Publication e Slide Show, e do lado direito são apresentadas algumas das propriedades que compõem a ontologia.
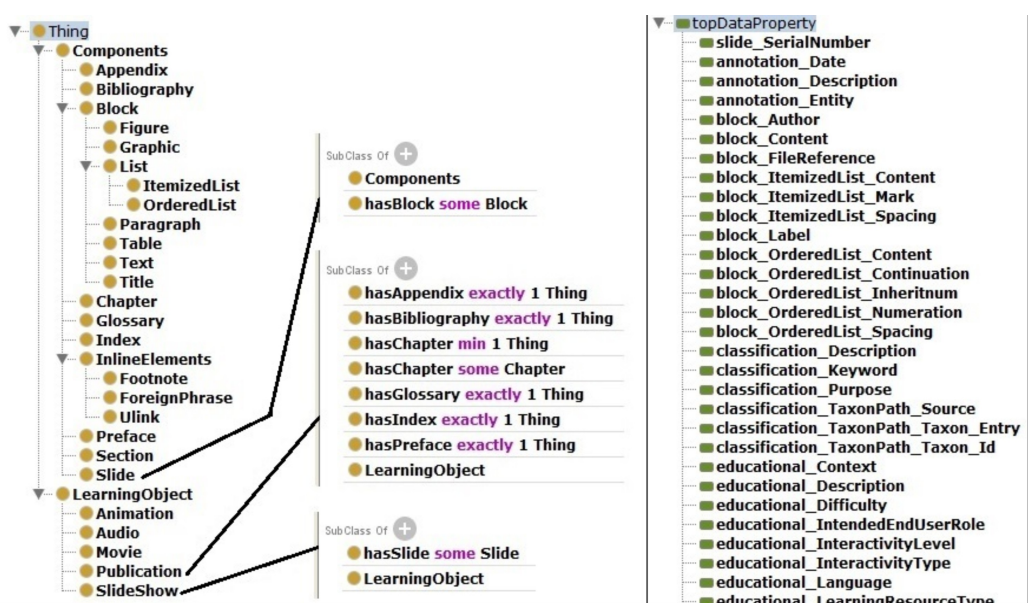

Figura 7. Ontologia ONTOER+ com suas restrições e propriedades de dados

O exemplo descrito a seguir ilustra o processo de criação de uma instância de um objeto de aprendizagem do tipo Slide Show. A Figura 8 apresenta os slides que compõem a apresentação de slides utilizada como exemplo e o Quadro 1 apresenta informações adicionais sobre a apresentação.

Quadro 1. Informações adicionais da apresentação em slides a ser instanciada

\begin{tabular}{|c|c|}
\hline 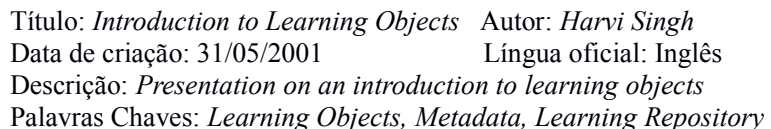 & $\begin{array}{l}\text { Versão atual: } 1.0 \quad \text { Status atual: finalized } \\
\text { Contribuição 1: Harvi Singh-author }-20 / 05 / 2001 \\
\text { Contribuição 2: Centra }- \text { collaborator }-25 / 05 / 2001 \\
\text { Formato: SlideShow } \quad \text { Tamanho em bytes: } 414720\end{array}$ \\
\hline
\end{tabular}

Na Figura 8, é possível observar objetos destacados no Slide 1 e no Slide 6. O Slide 1 possui os objetos Title e Text, enquanto que o Slide 6 possui os objetos Title, ItemizedList, Figure e Text. Para ilustrar o uso da ontologia, são considerados apenas estes dois slides (Slide 1 e Slide 6). Para que a apresentação em slides seja descrita pela ontologia, primeiro cria-se uma instância, que, no caso desse exemplo, denominamos 
"Introduction_To_Learning_Objects". As informações adicionais da apresentação em slides deverão ser relacionadas aos atributos de dados da ontologia que correspondem aos metadados do objeto de aprendizagem.

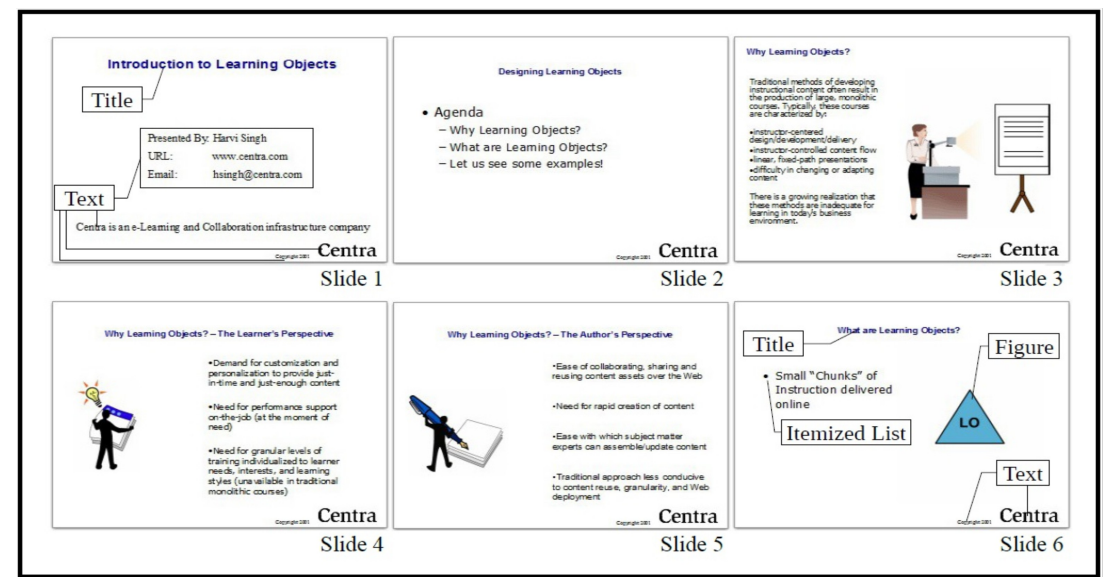

Figura 8. Apresentação em slides "Introduction to Learning Objects"

Em seguida, deve-se criar a relação entre os objetos de cada um dos slides e os atributos equivalentes na ontologia. A Figura 9 apresenta um exemplo de instância do tipo slide show. Foram considerados apenas o Slide 1 e o Slide 6, tendo em vista a repetição de objetos da apresentação nos demais slides, o que tornaria ilegível a leitura da representação da instância criada. As reticências indicam que existem slides no intervalo entre o Slide 1 e o Slide 6. "Introduction to Learning Objects" é um Learning Object do tipo Slide Show e possui components. Os dados adicionais, apresentados no Quadro 1 aparecem como metadados da instância criada. Os números 1 e 6 representam os slides instanciados que são componentes de uma apresentação. Observe que, cada slide possui blocos (classe block) onde o Slide 1 possui um elemento Title e quatro elementos Text e o Slide 6 possui um elemento Title, um elemento Figure, um elemento List e quatro elementos Text.

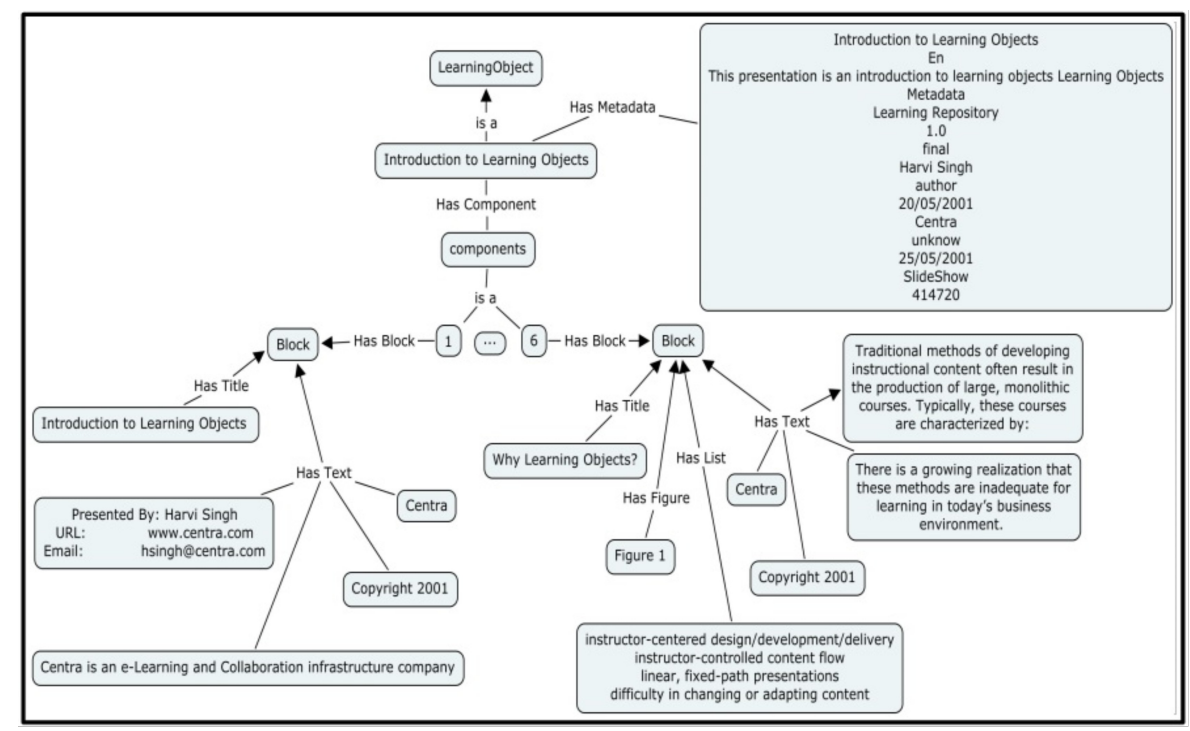

Figura 9. Instanciação do Slide 1 e do Slide 6 da apresentação "Introduction to Learning Objects" 


\section{CONCLUSÃO}

Este trabalho apresentou o processo de desenvolvimento da ontologia ONTOER+, que permite disponibilizar REA de forma fragmentada, contribuindo, dessa forma, para a reutilização de objetos de aprendizagem disponibilizados em formato aberto. A ontologia ONTOER+ é uma extensão da ontologia ONTOER, proposta em [Amorim et al. 2012], que oferece meios para a representação, de forma fragmentada, de REA do tipo livro. Como principais extensões implementadas na ONTOER+, destacam-se: i) a disponibilização de publicações (artigos, livros, apostilas e similares) de forma fragmentada: prefácio, índice, capítulo, seção, parágrafo, tabela, gráfico, figura, lista, nota de rodapé, palavras estrangeiras, listas de itens, apêndice e bibliografia; ii) a disponibilização de apresentações em slides de forma fragmentada: figura, tabela, itens, texto, título; iii) apesar de representar relação direta com os termos do DocBook, a ONTOER não apresenta as relações de restrições e cardinalidade exigidas para REA fragmentados; e iv) descrição de determinados conceitos de forma independente de hierarquia, por observar que nem todos os REA são fragmentados seguindo o mesmo padrão de um livro. Considerando, por exemplo, o conceito Block, um REA do tipo áudio tem blocos com características diferentes de um REA do tipo Publication.

Como metodologia de desenvolvimento da ontologia ONTOER + , foi utilizada a Methontology, com o intuito de facilitar o desenvolvimento da ontologia e também garantir uma documentação adequada das principais atividades e artefatos gerados. Como vantagens do uso da metodologia, identificou-se que as atividades iniciais de especificação e conceitualização auxiliam na produção de artefatos capazes de melhor conduzir a construção da ontologia desejada.

É importante ressaltar que este trabalho pode ser um ponto de partida para outras pesquisas. Eventuais problemas, tais como, uma fragmentação indesejada de um determinado recurso, não foram abordados aqui. Dessa forma, pesquisas mais aprofundadas, com o objetivo de solucionar tais situações, ainda são necessárias. Além disso, pesquisas podem ser realizadas no sentido de tratar problemas mais complexos, como a representação de REA obtidos a partir de um chat com texto e áudio em interações diversas, tais como em uma aula na modalidade a distância ou em uma entrevista.

\section{AGRADECIMENTOS}

Este trabalho foi apoiado pelo Instituto Nacional de Ciência e Tecnologia para Engenharia de Software (INES), financiado pelo CNPq e FACEPE, processos 573964/2008-4 e APQ-1037-1.03/08.

\section{REFERÊNCIAS}

Amorim, Ricardo; Rabelo, Thomas; Amorim, Dinani. (2012) Open Educational Resources Ontology. Em: Anais dos Workshops do Congresso Brasileiro de Informática na Educação, Rio de Janeiro. Disponível em http://www.brie.org/pub/index.php/ wcbie/article/view/1887/1651. Acesso em 08/01/2014.

Casali, A., Deco, C., Romano, A. \& Tomé, G. (2013) An Assistant for Loading Learning Object Metadata: an Ontology Based Approach. Interdisciplinary Journal of ELearning and Learning Objects IJELLO, v. 9, p. 11. 
Creative Commons. (2014) Get Creative Commons updates. Disponível em http://creativecommons.org/about. Acesso em 21/06/2014.

Corcho, Oscar; Fernández-López, Mariano; Gómez-Pérez, Asunción. (2003) Methodologies, tools and languages for building ontologies. Where is their meeting point?. Em: Data and Knowledge Engineering, v. 46, n. 1, p. 41-64.

Fernández-López, Mariano; Gómez-Pérez, Asunción; Juristo, Natalia. (1997) Methontology: from ontological art towards ontological engineering. Proc. AAAI Spring Symp. Series, AAAI Press, Menlo Park, Calif., pp. 33-40.

Fox, M. S. (1992) The TOVE Project: towards a common-sense model of the enterprise. Disponível em: $\quad<\mathrm{http}: / \mathrm{www}$.eil.utoronto.ca/enterprisemodelling/papers/index.html>. Acessado em: 16/07/2014.

Guimarães, Francisco José Zamith. (2002) Utilização de ontologias no domínio B2C. Mestrado em Informática, Pontifícia Universidade Católica, Rio de Janeiro. Disponível em: www2.dbd.pucrio.br/pergamum/tesesabertas/0024134_02_cap_04.pdf.

Hodgins, W. et al. (2002) Draft standard for learning object metadata. IEEE, v. 1484. Disponível em http://ltsc.ieee.org/wg12/files/LOM_1484_12_1_v1_Final_Draft.pdf. Acesso em 12/01/2014.

Mattos, Merisandra Côrtes de; Simões, Priscyla Waleska Targino de Azevedo; Farias, Renan Figueredo. (2010) A Metodologia Methontology na Construção de Ontologias. Revista de Iniciação Científica, v. 5, n. 1.

Park, Jinsoo; Sung, Kimoon; Moon, Sewon. (2008) Developing graduation screen ontology based on the METHONTOLOGY approach. In: Networked Computing and Advanced Information Management. p. 375-380.

Santana, B.; Rossini, C.; Pretto, N. (2012) Recursos Educacionais Abertos: práticas colaborativas e políticas públicas. Em: Recursos educacionais abertos: práticas colaborativas e políticas públicas. Edufba; Casa da Cultura Digital.

Santos, Andreia Inamorato dos. (2013) Recursos Educacionais Abertos no Brasil: o estado da arte, desafios e perspectivas para o desenvolvimento e inovação. São Paulo: Comitê Gestor da Internet no Brasil.

Silva, Daniela Lucas da; Souza, Renato Rocha; Almeida, Maurício Barcellos. (2008) Ontologias e vocabulários controlados: comparação de metodologias para construção. Ciência da Informação, Brasília, v. 37, n. 3, p. 60-75.

Suárez-Figueroa, Mari Carmen; Gómez-Pérez, Asunción; Fernández-López, Mariano. (2012) The NeOn methodology for ontology engineering. Em: Ontology engineering in a networked world. Springer Berlin Heidelberg. p. 9-34.

Walsh, Norman. (1999) DocBook: the definitive guide. "O'Reilly Media, Inc.". Disponível em: http://www.docbook.org/tdg/en/html/docbook.html. 